\title{
GLOBAL CONVERGENCE OF AN EFFICIENT HYBRID CONJUGATE GRADIENT METHOD FOR UNCONSTRAINED OPTIMIZATION
}

\author{
Jinkui LiU AND Xianglin Du
}

\begin{abstract}
In this paper, an efficient hybrid nonlinear conjugate gradient method is proposed to solve general unconstrained optimization problems on the basis of CD method [2] and DY method [5], which possess the following property: the sufficient descent property holds without any line search. Under the Wolfe line search conditions, we proved the global convergence of the hybrid method for general nonconvex functions. The numerical results show that the hybrid method is especially efficient for the given test problems, and it can be widely used in scientific and engineering computation.
\end{abstract}

\section{Introduction}

The primary objective of this paper is to study the global convergence property and practical computational performance of a new hybrid conjugate gradient method with the Wolfe line search for nonlinear unconstrained optimization.

Consider the following unconstrained optimization problem

$$
\min _{x \in \mathbb{R}^{n}} f(x),
$$

where $f: \mathbb{R}^{n} \rightarrow \mathbb{R}$ is smooth and its gradient is available. The conjugate gradient method is very useful for solving (1.1) especially when $n$ is large, and has the following iterative formulas:

$$
\begin{gathered}
x_{k+1}=x_{k}+\alpha_{k} d_{k}, \\
d_{k}= \begin{cases}-g_{k}, & k=1, \\
-g_{k}+\beta_{k} d_{k-1}, & k \geq 2,\end{cases}
\end{gathered}
$$

where $x_{k}$ is the current iteration point, $g_{k}$ is the gradient of $f$ at $x_{k}, \alpha_{k}$ is a positive scalar and called the steplength which is determined by some line

Received April 1, 2011; Revised April 19, 2012.

2010 Mathematics Subject Classification. 46N10.

Key words and phrases. unconstrained optimization, conjugate gradient method, the Wolfe line search, descent property, global convergence.

This work was supported by the Nature Science Foundation of Chongqing Education Committee (KJ121112). 
search, $d_{k}$ is the search direction, and $\beta_{k}$ is a scalar. There are many ways to select $\beta_{k}$, and some well-known formulas are given by

$$
\begin{aligned}
\beta_{k}^{P R P} & =\frac{g_{k}^{T} y_{k-1}}{\left\|g_{k-1}\right\|^{2}}(\text { Polak-Ribiere-Polak [9], [8]), } \\
\beta_{k}^{D Y} & =\frac{\left\|g_{k}\right\|^{2}}{d_{k-1}^{T} y_{k-1}}(\text { Dai-Yuan }[2]), \\
\beta_{k}^{C D} & =-\frac{\left\|g_{k}\right\|^{2}}{d_{k-1}^{T} g_{k-1}}(\text { Fletcher }[5]),
\end{aligned}
$$

respectively, where $\|\cdot\|$ is the Euclidean norm and $y_{k-1}=g_{k}-g_{k-1}$. The corresponding methods are called the PRP method, DY method and CD method, respectively.

In the convergence analysis and implementations of conjugate gradient methods, one often requires the line search to satisfy the strong Wolfe line search conditions, namely

$$
\begin{gathered}
f\left(x_{k}+\alpha_{k} d_{k}\right)-f\left(x_{k}\right) \leq \delta \alpha_{k} g_{k}^{T} d_{k}, \\
\left|g\left(x_{k}+\alpha_{k} d_{k}\right)^{T} d_{k}\right| \leq-\sigma g_{k}^{T} d_{k},
\end{gathered}
$$

where $0<\delta<\sigma<1$. The PRP method is regarded as the best one in practical computation. However, the PRP method has no global convergence in some situations. So some people have studied modified PRP methods. For example, Gilbert and Nocedal [6] proved that the conjugate gradient method with $\beta_{k}=$ $\max \left\{\beta_{k}^{P R P}, 0\right\}$ converged globally (The corresponding method is the famous $\mathrm{PRP}^{+}$method), where the strong Wolfe line search and the sufficient descent condition were satisfied. Dai and Yuan [2] proved that the DY method could produce a descent search direction at every iteration and converge globally, where the line search satisfied the Wolfe line search conditions, namely, (1.7) and

$$
g\left(x_{k}+\alpha_{k} d_{k}\right)^{T} d_{k}>\sigma g_{k}^{T} d_{k},
$$

where $0<\delta<\sigma<1$. Dai and Yuan [4] proved the CD method could ensure all search direction downhill, as long as the strong Wolfe line search conditions were satisfied. Dai [1] proposed a new conjugate gradient method in which

$$
\beta_{k}=\frac{\left\|g_{k}\right\|^{2}}{\max \left\{d_{k-1}^{T} y_{k-1},-d_{k-1}^{T} g_{k-1}\right\}},
$$

and proved the global convergence under the nonmonotone line search. Wei, Yao and Liu [10] gave a new conjugate gradient method where the parameter $\beta_{k}$ satisfies the following formula:

$$
\beta_{k}^{V P R P}=\frac{g_{k}\left(g_{k}-\frac{\left\|g_{k}\right\|}{\left\|g_{k-1}\right\|} g_{k-1}\right)}{\left\|g_{k-1}\right\|^{2}} .
$$


They discussed the global convergence of the VPRP under the exact line search, the Wolfe line search and the Grippo Lucidi line search, respectively. The corresponding method is called the VPRP method in this paper.

The aim of this paper is to choose $\beta_{k}$ to ensure that $d_{k}$ is a descent direction and, at the same time, ensure the global convergence.

Under the inexact line searches, based on the effectively global convergence of the DY method and the well descent property of the CD method, we choose $\beta_{k}$ to satisfy:

$$
\beta_{k}=\left\{\begin{array}{l}
0, \quad \text { if } d_{k-1}^{T} g_{k} \leq \sigma d_{k-1}^{T} g_{k-1} \\
\beta_{k}^{C D}, \quad \text { if } \sigma d_{k-1}^{T} g_{k-1}<d_{k-1}^{T} g_{k} \leq 0 \\
\beta_{k}^{D Y}, \quad \text { if } 0<d_{k-1}^{T} g_{k}<\mu d_{k-1}^{T}\left(g_{k}-g_{k-1}\right), \\
\mu\left\|g_{k}\right\|^{2} / d_{k-1}^{T} g_{k}, \quad \text { if } d_{k-1}^{T} g_{k} \geq \mu d_{k-1}^{T}\left(g_{k}-g_{k-1}\right),
\end{array}\right.
$$

where $0<\mu \leq \sigma$. In this paper, the corresponding descent method is called as CDY method, and we can prove that the CDY method has the sufficient descent property and the global convergence property.

\section{The descent property}

In order to prove the global convergence of the CDY method, the objective function $f(x)$ satisfies the following assumption.

\section{Assumption (H):}

(1) $f(x)$ is bounded from below on the level set $\Omega=\left\{x \mid f(x) \leq f\left(x_{1}\right)\right\}$.

(2) The objective function $f(x)$ is continuously differentiable, and its gradient $g(x)$ is Lipschitz continuous on the open set $\Gamma$ containing $\Omega$, i.e., there exists a constant $L>0$ such that

$$
\|g(x)-g(y)\| \leq L\|x-y\| \text { for all } x, y \in \Gamma .
$$

\section{CDY method:}

Data: $x_{1} \in \mathbb{R}^{n}, \varepsilon \geq 0$.

Step 1: Set $d_{1}=-g_{1}$, if $\left\|g_{1}\right\| \leq \varepsilon$, then stop.

Step 2: Compute $\alpha_{k}$ by some inexact line search.

Step 3: Let $x_{k+1}=x_{k}+\alpha_{k} d_{k}, g_{k+1}=g\left(x_{k+1}\right)$, if $\left\|g_{k+1}\right\| \leq \varepsilon$, then stop.

Step 4: Compute $\beta_{k+1}$ by (1.10), and generate $d_{k+1}$ by (1.3).

Step 5: Set $k=k+1$, go to Step 2 .

In most references, we can see that the sufficient descent condition

$$
g_{k}^{T} d_{k} \leq-c\left\|g_{k}\right\|^{2}, c>0
$$

is always given which plays a vital role in guaranteeing the global convergence properties of conjugate gradient methods. Furthermore, we have the following lemma which illustrates that the CDY method has the sufficient property for any line search. 
Lemma 1. Consider the iteration (1.2)-(1.3), where $\alpha_{k}$ is computed by any inexact line search, and $\beta_{k}$ satisfies (1.10). Then if $g_{k} \neq 0$ for $k \geq 1$, we have that

$$
g_{k}^{T} d_{k} \leq-\left\|g_{k}\right\|^{2} \quad \text { for } \forall \geq 1 .
$$

Proof. To obtain this result, we divide the proof into four aspects as follows. have

(i) If $d_{k-1}^{T} g_{k} \leq \sigma g_{k-1}^{T} d_{k-1}$, we get $\beta_{k}=0$. By multiplying (1.3) with $g_{k}$, we

$$
g_{k}^{T} d_{k}=-\left\|g_{k}\right\|^{2}+\beta_{k} g_{k}^{T} d_{k-1} .
$$

Obviously, $g_{k}^{T} d_{k}=-\left\|g_{k}\right\|^{2}<0$.

(ii) If $\sigma g_{k-1}^{T} d_{k-1}<g_{k}^{T} d_{k-1} \leq 0$, then $\beta_{k}=\beta_{k}^{C D}$. From (1.6), we have $\beta_{k}=\beta_{k}^{C D}>0$. From (2.3), we easily have $g_{k}^{T} d_{k} \leq-\left\|g_{k}\right\|^{2}<0$.

(iii) If $0<g_{k}^{T} d_{k-1}<\mu d_{k-1}^{T}\left(g_{k}-g_{k-1}\right)$, we have $\beta_{k}=\beta_{k}^{D Y}$. Then from (2.3) and (1.5), we have

$$
g_{k}^{T} d_{k}=-\left\|g_{k}\right\|^{2}+\frac{\left\|g_{k}\right\|^{2}}{d_{k-1}^{T}\left(g_{k}-g_{k-1}\right)} \cdot d_{k-1}^{T} g_{k} .
$$

From (2.4) and $0<g_{k}^{T} d_{k-1}<\mu d_{k-1}^{T}\left(g_{k}-g_{k-1}\right)$, we have

$$
g_{k}^{T} d_{k}<-\left\|g_{k}\right\|^{2}+\frac{\left\|g_{k}\right\|^{2}}{\frac{1}{\mu} \cdot d_{k-1}^{T} g_{k}} \cdot d_{k-1}^{T} g_{k}=-(1-\mu)\left\|g_{k}\right\|^{2}<0 .
$$

(iv) If $g_{k}^{T} d_{k-1} \geq \mu d_{k-1}^{T}\left(g_{k}-g_{k-1}\right)$, we have $\beta_{k}=\mu\left\|g_{k}\right\|^{2} / d_{k-1}^{T} g_{k}$. Then from (2.3), we have

$$
g_{k}^{T} d_{k}=-\left\|g_{k}\right\|^{2}+\mu\left\|g_{k}\right\|^{2}=-(1-\mu)\left\|g_{k}\right\|^{2} \leq-(1-\sigma)\left\|g_{k}\right\|^{2}<0 .
$$

This completes the proof of Lemma 1.

\section{Global convergence}

In this section, we will study the global convergence of the CDY method with the Wolfe line search. The following lemma, often called the Zoutendijk condition, is used to prove the global convergence of nonlinear conjugate gradient methods. It was originally given by Zoutendijk [11].

Lemma 2. Suppose Assumption (H) holds. Consider any iteration of the form (1.2)-(1.3), where $d_{k}$ satisfies $d_{k}^{T} g_{k}<0$ for $k \in \mathbb{N}$ and $\alpha_{k}$ satisfies the Wolfe line search (1.7) and (1.9). Then

$$
\sum_{k \geq 1} \frac{\left(g_{k}^{T} d_{k}\right)^{2}}{\left\|d_{k}\right\|^{2}}<+\infty
$$

Theorem 3. Suppose that Assumption $(\mathrm{H})$ holds. Consider any iteration of the form (1.2)-(1.3), where $\beta_{k}$ is computed by (1.10) and $\alpha_{k}$ satisfies the Wolfe line search (1.7) and (1.9). Then we have either $g_{k}=0$ for some $k$, or

$$
\liminf _{k \rightarrow+\infty}\left\|g_{k}\right\|=0 .
$$


Proof. If $g_{k}=0$ holds for some finite $k$, then we know that $x_{k}$ is a stationary point. Otherwise, we prove the conclusion (3.2) by contradiction.

Suppose that (3.2) dose not hold. This means that the gradients remain bounded away from zero, and hence there exists $\gamma>0$ such that

$$
\left\|g_{k}\right\| \geq \gamma \text { for } \forall k \geq 1 \text {. }
$$

From (1.9) and (2.2), we have

$$
d_{k-1}^{T}\left(g_{k}-g_{k-1}\right) \geq-(1-\sigma) d_{k-1}^{T} g_{k-1}>0 .
$$

In the following, we will prove that $\beta_{k} \leq \beta_{k}^{D Y}$ holds, for $\forall k \geq 1$.

First, if $\sigma g_{k-1}^{T} d_{k-1} \leq g_{k}^{T} d_{k-1} \leq 0$, then we have $\beta_{k}=\beta_{k}^{C D}$ and

$$
\begin{aligned}
-g_{k-1}^{T} d_{k-1} & \geq-g_{k-1}^{T} d_{k-1}+g_{k}^{T} d_{k-1} \\
& \geq-(1-\sigma) d_{k-1}^{T} g_{k-1} .
\end{aligned}
$$

From (1.5), (1.6) and (3.5), we have

$$
\beta_{k}=\beta_{k}^{C D} \leq \beta_{k}^{D Y} .
$$

Second, if $0<g_{k}^{T} d_{k-1}<\mu d_{k-1}^{T}\left(g_{k}-g_{k-1}\right)$, then form (1.10), we have

$$
\beta_{k}=\beta_{k}^{D Y} \text {. }
$$

Third, if $g_{k}^{T} d_{k-1} \geq \mu d_{k-1}^{T}\left(g_{k}-g_{k-1}\right)$, from (3.4), we have $\mu d_{k-1}^{T}\left(g_{k}-g_{k-1}\right)>0$. Then

$$
\beta_{k}=\mu\left\|g_{k}\right\|^{2} / d_{k-1}^{T} g_{k} \leq \beta_{k}^{D Y} .
$$

Y. H. Dai and Y. Yuan [3] gives an equivalent formula to (1.5):

$$
\beta_{k}^{D Y}=\frac{g_{k}^{T} d_{k}}{g_{k-1}^{T} d_{k-1}} .
$$

What's more, if $d_{k-1}^{T} g_{k}<\sigma g_{k-1}^{T} d_{k-1}$, we have $\beta_{k}=0$. So from (3.6)-(3.9), we can get

$$
\beta_{k} \leq \frac{g_{k}^{T} d_{k}}{g_{k-1}^{T} d_{k-1}} \text { for } \forall k \geq 1
$$

On the one hand, writing (1.3) as $d_{k}+g_{k}=\beta_{k} d_{k-1}$ and squaring it, we get

$$
\left\|d_{k}\right\|^{2}=-\left\|g_{k}\right\|^{2}-2 g_{k}^{T} d_{k}+\left(\beta_{k}\right)^{2}\left\|d_{k-1}\right\|^{2} .
$$

From (3.10), we have

$$
\left\|d_{k}\right\|^{2} \leq-\left\|g_{k}\right\|^{2}-2 g_{k}^{T} d_{k}+\left(\frac{g_{k}^{T} d_{k}}{g_{k-1}^{T} d_{k-1}}\right)^{2}\left\|d_{k-1}\right\|^{2} .
$$


Dividing above inequality by $\left(g_{k}^{T} d_{k}\right)^{2}$, we have

$$
\begin{aligned}
\frac{\left\|d_{k}\right\|^{2}}{\left(g_{k}^{T} d_{k}\right)^{2}} & \leq \frac{\left\|d_{k-1}\right\|^{2}}{\left(g_{k-1}^{T} d_{k-1}\right)^{2}}-\left(\frac{\left\|g_{k}\right\|}{g_{k}^{T} d_{k}}+\frac{1}{\left\|g_{k}\right\|}\right)^{2}+\frac{1}{\left\|g_{k}\right\|^{2}} \\
& \leq \frac{\left\|d_{k-1}\right\|^{2}}{\left(g_{k-1}^{T} d_{k-1}\right)^{2}}+\frac{1}{\left\|g_{k}\right\|^{2}} .
\end{aligned}
$$

Using (3.11) recursively and noting that $\left\|d_{1}\right\|^{2}=-g_{1}^{T} d_{1}=\left\|g_{1}\right\|^{2}$, we get

$$
\frac{\left\|d_{k}\right\|^{2}}{\left(g_{k}^{T} d_{k}\right)^{2}} \leq \sum_{i=1}^{k} \frac{1}{\left\|g_{k}\right\|^{2}} \text {. }
$$

Then we get from this and (3.3) that

$$
\frac{\left(g_{k}^{T} d_{k}\right)^{2}}{\left\|d_{k}\right\|^{2}} \geq \frac{\gamma^{2}}{k}
$$

which indicates

$$
\sum_{k \geq 1} \frac{\left(g_{k}^{T} d_{k}\right)^{2}}{\left\|d_{k}\right\|^{2}}=+\infty .
$$

This contradicts the Zoutendijk condition (3.1). Therefore the conclusion (3.2) holds.

Corollary. Suppose that Assumption (H) holds. Consider any iteration of the form (1.2)-(1.3), where $\beta_{k}$ is computed by (1.10) and $\alpha_{k}$ satisfies the strong Wolfe line search (1.7)-(1.8). Then we have either $g_{k}=0$ for some $k$, or

$$
\liminf _{k \rightarrow+\infty}\left\|g_{k}\right\|=0 \text {. }
$$

Proof. If $\alpha_{k}$ satisfies the strong Wolfe line search (1.7)-(1.8), then $\alpha_{k}$ must satisfy the Wolfe line search (1.7) and (1.9). Therefore the statement follows Theorem 3.

\section{Numerical results}

The purpose of this section is to present computational supports for the CD method, DY method, $\mathrm{PRP}^{+}$method, VPRP method and $\operatorname{CDY}\left(\mu=10^{-6}\right)$ method. Under the strong Wolfe line searches, the methods were tested for a set of standard unconstrained minimization test problems from [7], where $\delta=0.01, \sigma=0.1$. The termination condition of the experiments is $\left\|g_{k}\right\| \leq \varepsilon$, where $\varepsilon=10^{-6}$.

The test problems are listed in Table 1, and the detail numerical results of our tests are reported in Tables 2-4. In Tables, "Number" and "Name" denote the problem number and problem name, respectively. "Dim" denotes the dimension of the test problems. "__ means the method fails. In Table 2 , the detailed numerical results are listed in the form NI/NF/NG, where NI, $\mathrm{NF}$, NG denote the number of iterations, function evaluations, and gradient 
TABLE 1. List of test problems

\begin{tabular}{|c|c|}
\hline Number & Name \\
1 & Freudenstein and Roth function \\
2 & Beale function \\
3 & Helical valley function \\
4 & Gulf research and development function \\
5 & Powell singular function \\
6 & Wood function \\
7 & Kowalik and Osborne function \\
8 & Brown and Dennis function \\
9 & Watson function \\
10 & Penalty function I \\
11 & Trigonometric function \\
12 & Extended Powell singular function \\
13 & Discrete boundary value function \\
14 & Discrete integral equation function \\
15 & Broyden tridiagonal function \\
\hline
\end{tabular}

TABLE 2. The numerical results of the methods

\begin{tabular}{|c|c|c|c|c|c|c|}
\hline Number & Dim & CD & DY & PRP & VPRP & CDY \\
\hline 1 & 2 & $51 / 187 / 152$ & $42 / 168 / 138$ & $11 / 72 / 56^{*}$ & $15 / 90 / 70$ & $11 / 76 / 57$ \\
2 & 2 & $73 / 177 / 155$ & $75 / 186 / 164$ & $13 / 58 / 45^{*}$ & $21 / 65 / 47$ & $15 / 57 / 44$ \\
3 & 3 & $56 / 157 / 132$ & $37 / 118 / 98^{*}$ & $65 / 181 / 156$ & $57 / 174 / 146$ & $45 / 147 / 123$ \\
4 & 3 & $1 / 2 / 2^{*}$ & $1 / 2 / 2^{*}$ & $1 / 2 / 2^{*}$ & $1 / 2 / 2^{*}$ & $1 / 2 / 2^{*}$ \\
5 & 4 & $421 / 1036 / 947$ & $2286 / 4555 / 4545$ & $113 / 379 / 328$ & $87 / 300 / 253^{*}$ & $102 / 383 / 333$ \\
6 & 4 & $184 / 438 / 399$ & $100 / 291 / 240$ & $118 / 357 / 304$ & $236 / 608 / 543$ & $78 / 278 / 230^{*}$ \\
7 & 4 & $254 / 723 / 633$ & $536 / 1449 / 1271$ & $93 / 269 / 240$ & $66 / 228 / 197^{*}$ & $68 / 249 / 220$ \\
8 & 4 & $44 / 171 / 133$ & $39 / 158 / 121$ & $37 / 156 / 123^{*}$ & & $41 / 178 / 136$ \\
9 & 5 & $87 / 277 / 239^{*}$ & $127 / 348 / 299$ & $133 / 374 / 330$ & $130 / 377 / 324$ & $402 / 1208 / 1062$ \\
& 15 & $4377 / 12694 / 11221$ & $1845 / 5658 / 4924$ & $3290 / 10457 / 9244$ & $1800 / 5631 / 4954^{*}$ & $2288 / 7973 / 7044$ \\
10 & 100 & $62 / 223 / 182$ & $31 / 157 / 121$ & $29 / 168 / 128^{*}$ & $34 / 224 / 170$ & $33 / 195 / 152$ \\
& 200 & $25 / 159 / 117$ & $26 / 160 / 121$ & $25 / 175 / 132$ & $34 / 239 / 181$ & $24 / 167 / 125^{*}$ \\
11 & 100 & - & $306 / 401 / 400$ & $58 / 120 / 113$ & $56 / 145 / 119$ & $56 / 137 / 121^{*}$ \\
& 200 & & $315 / 399 / 398$ & $64 / 135 / 128$ & $58 / 126 / 122^{*}$ & $64 / 160 / 146$ \\
12 & 500 & $748 / 1780 / 1689$ & $2778 / 5384 / 5374$ & $105 / 342 / 297$ & $178 / 612 / 526$ & $100 / 379 / 324^{*}$ \\
& 1000 & $578 / 1419 / 1292$ & $4329 / 8191 / 18168$ & $198 / 693 / 595$ & $106 / 355 / 305^{*}$ & $154 / 623 / 552$ \\
13 & 500 & $5089 / 7049 / 7048$ & $4796 / 6823 / 6822$ & $1645 / 2889 / 2888$ & $1461 / 2534 / 2533$ & $219 / 491 / 466^{*}$ \\
& 1000 & $2406 / 3114 / 3113$ & $414 / 449 / 448$ & $147 / 251 / 250$ & $157 / 275 / 274$ & $38 / 69 / 64^{*}$ \\
14 & 500 & $7 / 15 / 8$ & $7 / 15 / 8$ & $6 / 13 / 7^{*}$ & $7 / 15 / 8$ & $7 / 15 / 8$ \\
& 1000 & $7 / 15 / 8$ & $7 / 15 / 8$ & $6 / 13 / 7^{*}$ & $7 / 15 / 8$ & $7 / 15 / 8$ \\
15 & 500 & $52 / 112 / 107$ & $49 / 106 / 101$ & $35 / 78 / 74$ & $35 / 78 / 73$ & $33 / 75 / 60^{*}$ \\
& 1000 & $70 / 149 / 145$ & $64 / 137 / 133$ & $34 / 76 / 72$ & $36 / 81 / 77$ & $32 / 75 / 61^{*}$ \\
\hline
\end{tabular}

evaluations, respectively. The star "*" denotes that this result is best one among these methods. In Table 3, CPU times of the methods are given. The final values and standard values are reported in Table 4. "Standard" means standard value of the test problem.

Tables 2-3 show that the CDY method has the best performance with respect to the number of iterations and the CPU time. What is more, Table 4 also show that the CDY method relative to the final values of the test problems is 
comparable with that of the PRP + method and VPRP method. All numerical results show that the efficiency of the CDY method is encouraging.

TABLE 3. The corresponding CPU times of the methods

\begin{tabular}{|c|c|c|c|c|c|c|}
\hline Number & Dim & CD & DY & PRP $^{+}$ & VPRP & CDY \\
\hline 1 & 2 & 0.1863 & 0.1675 & 0.0510 & 0.0494 & $0.0452^{*}$ \\
2 & 2 & 0.2517 & 0.3000 & $0.0714^{*}$ & 0.0735 & 0.0720 \\
3 & 3 & 0.1985 & $0.1263^{*}$ & 0.4376 & 0.1982 & 0.2531 \\
4 & 3 & 0.0036 & 0.0037 & 0.0033 & 0.0034 & $0.0009^{*}$ \\
5 & 4 & 2.5067 & 8.9351 & 0.7034 & $0.3132^{*}$ & 0.3892 \\
6 & 4 & 0.7156 & 0.4189 & 0.5263 & 1.2076 & $0.3787^{*}$ \\
7 & 4 & 0.6112 & 2.1098 & 0.4172 & $0.3289^{*}$ & 0.3579 \\
8 & 4 & $0.1614^{*}$ & 0.3521 & 0.2172 & - & 0.2364 \\
9 & 5 & $0.3142^{*}$ & 0.5312 & 0.6102 & 0.5762 & 2.2067 \\
& 15 & 16.9905 & $6.0000^{*}$ & 10.9487 & 6.8933 & 7.5893 \\
10 & 100 & 0.5118 & 0.2325 & 0.2712 & 0.3102 & $0.2136^{*}$ \\
& 200 & 0.3952 & 0.4015 & 0.4306 & 0.6212 & $0.3657^{*}$ \\
11 & 100 & - & 1.9000 & 0.4573 & 0.4384 & $0.3899^{*}$ \\
& 200 & - & 6.5000 & $1.8896^{*}$ & 1.9796 & 1.9860 \\
12 & 500 & 12.9834 & 32.8723 & 2.4000 & 3.5617 & $2.2670^{*}$ \\
& 1000 & 28.7000 & 43.2000 & 7.3000 & $6.7891^{*}$ & 11.2701 \\
13 & 500 & 58.1386 & 55.4843 & 27.8092 & 21.1132 & $8.7861^{*}$ \\
& 1000 & 39.6372 & 13.0000 & 6.1000 & 7.2000 & $1.8963^{*}$ \\
14 & 500 & 2.1428 & 2.1554 & $1.8556^{*}$ & 2.1450 & 1.9325 \\
& 1000 & 8.5477 & 9.7059 & $7.3761^{*}$ & 8.5380 & 8.3450 \\
15 & 500 & 0.7284 & 0.6892 & 0.5063 & 0.5092 & $0.5011^{*}$ \\
& 1000 & 3.6011 & 3.1574 & 1.6478 & 1.7671 & $1.4968^{*}$ \\
\hline
\end{tabular}

TABLE 4. The final values and standard values of the methods

\begin{tabular}{|c|c|c|c|c|c|c|c|}
\hline Number & Dim & $\mathrm{CD}$ & $\overline{D Y}$ & $\mathrm{PRP}^{+}$ & VPRP & CDY & Standard \\
\hline 1 & 2 & $48.9843^{*}$ & $48.9843^{*}$ & $48.9843^{*}$ & $48.9843^{*}$ & $48.9843^{*}$ & 48.9842 \\
\hline 2 & 2 & $2.0949 \mathrm{e}-013$ & $7.5278 \mathrm{e}-014$ & $5.7209 \mathrm{e}-016$ & $3.1303 \mathrm{e}-019^{*}$ & $3.4197 \mathrm{e}-014$ & 0 \\
\hline 3 & 3 & $1.7392 \mathrm{e}-015^{*}$ & $1.2045 \mathrm{e}-014$ & $7.5337 \mathrm{e}-015$ & $5.2175 \mathrm{e}-015$ & $4.5381 \mathrm{e}-015$ & 0 \\
\hline 4 & 3 & $0.0385^{*}$ & $0.0385^{*}$ & $0.0385^{*}$ & $0.0385^{*}$ & $0.0385^{*}$ & 0 \\
\hline 5 & 4 & $1.7630 \mathrm{e}-010$ & $2.8606 \mathrm{e}-011$ & $1.4371 \mathrm{e}-012^{*}$ & $5.2126 \mathrm{e}-010$ & $2.0535 \mathrm{e}-011$ & 0 \\
\hline 6 & 4 & $4.6149 \mathrm{e}-014$ & $2.6023 \mathrm{e}-014^{*}$ & $3.0109 \mathrm{e}-013$ & $2.6463 \mathrm{e}-014$ & $8.6269 \mathrm{e}-014$ & 0 \\
\hline 7 & 4 & $3.0751 \mathrm{e}-004^{*}$ & $3.0751 \mathrm{e}-004^{*}$ & $3.0751 \mathrm{e}-004^{*}$ & $3.0751 \mathrm{e}-004^{*}$ & $3.0751 \mathrm{e}-004^{*}$ & $3.07505 \mathrm{e}-004$ \\
\hline 8 & 4 & $8.5822 \mathrm{e}+004^{*}$ & $8.5822 \mathrm{e}+004^{*}$ & $8.5822 \mathrm{e}+004^{*}$ & & $8.5822 \mathrm{e}+004^{*}$ & $8.5822 \mathrm{e}+004$ \\
\hline \multirow[t]{2}{*}{ 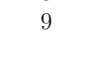 } & 5 & $0.0172^{*}$ & $0.0172^{*}$ & $0.0172^{*}$ & $0.0172^{*}$ & $0.0172^{*}$ & 0.0172 \\
\hline & 15 & $1.6303 \mathrm{e}-009$ & $2.8710 \mathrm{e}-010$ & $3.4585 \mathrm{e}-008$ & $3.7954 \mathrm{e}-008$ & $4.0916 \mathrm{e}-010^{*}$ & $4.7224 \mathrm{e}-010$ \\
\hline \multirow[t]{2}{*}{10} & 100 & $9.0249 \mathrm{e}-004^{*}$ & $9.0249 \mathrm{e}-004^{*}$ & $9.0249 \mathrm{e}-004^{*}$ & $9.0249 \mathrm{e}-004^{*}$ & $9.0249 \mathrm{e}-004^{*}$ & $9.0249 \mathrm{e}-004$ \\
\hline & 200 & $0.0019^{*}$ & $0.0019^{*}$ & $0.0019^{*}$ & $0.0019^{*}$ & $0.0019^{*}$ & 0.0019 \\
\hline \multirow[t]{2}{*}{11} & 100 & & $1.8410 \mathrm{e}-006^{*}$ & $1.8410 \mathrm{e}-006^{*}$ & $1.8410 \mathrm{e}-006^{*}$ & $1.8410 \mathrm{e}-006^{*}$ & 0 \\
\hline & 200 & & $1.0051 \mathrm{e}-006^{*}$ & $1.1542 \mathrm{e}-006$ & $1.1542 \mathrm{e}-006$ & $1.1542 \mathrm{e}-006$ & 0 \\
\hline \multirow[t]{2}{*}{12} & 500 & $4.4691 \mathrm{e}-010$ & $3.1934 \mathrm{e}-010$ & $4.3989 \mathrm{e}-011^{*}$ & $7.3238 \mathrm{e}-010$ & $7.8245 \mathrm{e}-010$ & 0 \\
\hline & 1000 & $9.9418 \mathrm{e}-010$ & $4.5246 \mathrm{e}-010$ & $1.2283 \mathrm{e}-010$ & $5.9312 \mathrm{e}-011^{*}$ & $6.2169 \mathrm{e}-011$ & 0 \\
\hline \multirow[t]{2}{*}{13} & 500 & $9.2306 \mathrm{e}-009$ & 7.4621e-009 & $8.9750 \mathrm{e}-009$ & $9.0694 \mathrm{e}-009$ & $1.0275 \mathrm{e}-009^{*}$ & 0 \\
\hline & 1000 & $1.2344 \mathrm{e}-009^{*}$ & $1.2373 \mathrm{e}-009$ & $1.2644 \mathrm{e}-009$ & $1.2616 \mathrm{e}-009$ & $1.2926 \mathrm{e}-009$ & 0 \\
\hline \multirow[t]{2}{*}{14} & 500 & $1.0452 \mathrm{e}-013$ & $1.1936 \mathrm{e}-013$ & $1.4385 \mathrm{e}-015$ & $1.2338 \mathrm{e}-015^{*}$ & $1.1623 \mathrm{e}-014$ & 0 \\
\hline & 1000 & $2.0882 \mathrm{e}-013$ & $5.7282 \mathrm{e}-015$ & $2.8738 \mathrm{e}-015$ & $2.4651 \mathrm{e}-015^{*}$ & $2.3219 \mathrm{e}-014$ & 0 \\
\hline \multirow[t]{2}{*}{15} & 500 & $1.3526 \mathrm{e}-014$ & $1.1159 \mathrm{e}-014$ & $6.6559 \mathrm{e}-015^{*}$ & $7.4750 \mathrm{e}-015$ & $7.1464 \mathrm{e}-015$ & 0 \\
\hline & 1000 & $9.6485 \mathrm{e}-015$ & $1.0529 \mathrm{e}-014$ & $1.1090 \mathrm{e}-014$ & $4.4833 \mathrm{e}-015$ & $3.9079 \mathrm{e}-015^{*}$ & 0 \\
\hline
\end{tabular}




\section{References}

[1] Y. H. Dai, A nonmonotone conjugate gradient algorithm for unconstrained optimization, J. Syst. Sci. Complex. 15 (2002), no. 2, 139-145.

[2] Y. H. Dai and Y. X. Yuan, A Nonlinear conjugate gradient with a strong global convergence property, SIAM. J. Optimization 10 (2000), 177-182.

[3] - Nonlinear Conjugate Gradient Method, Shanghai Scientific and Technical, Shanghai, China, 2000.

[4] - Convergence properties of the conjugate descent method, Adv. in Math. (China) 25 (1996), no. 6, 552-562.

[5] R. Fletcher, Practical Methods of Optimization, John Wiley \& Sons, Ltd., 1987.

[6] J. C. Gilbert and J. Nocedal, Global convergence properties of conjugate gradient methods for optimization, SIAM J. Optim. (1992), no. 1, 21-42.

[7] J. J. More, B. S. Garbow, and K. E. Hillstrome, Testing unconstrained optimization software, ACM Trans. Math. Software 7 (1981), no. 1, 17-41.

[8] B. T. Polak, The conjugate gradient method in extreme problems, USSR Comput. Math. Math. Phys. 9 (1969), 94-112.

[9] E. Polak and G. Ribiére, Note sur la convergence de méthodes de directions conjuguées, Rev. Française Informat. Recherche Opérationnelle 3 (1969) no. 16, 35-43.

[10] Z. Wei, S. Yao, and L. Liu, The convergence properties of some new conjugate gradient methods, Appl. Math. Comput. 183 (2006), no. 2, 1341-1350.

[11] G. Zoutendijk, Nonlinear Programming Computational Methods, Integer and nonlinear programming, pp. 37-86. North-Holland, Amsterdam, 1970.

JINKUI LIU

School of Mathematics and Statistics

Chongqing Three Gorges University

Chongqing, P. R. China

E-mail address: liujinkui2006@126.com

XiAngLin Du

School of Mathematics and Statistics

Chongqing Three Gorges University

Chongqing, P. R. China

E-mail address: duxianglin2002@126.com 